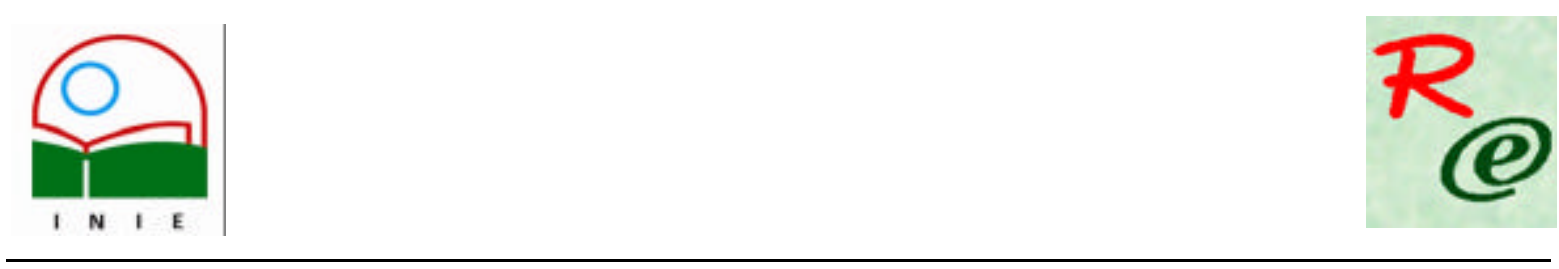

Actualidades Investigativas en Educación

Revista Electrónica publicada por el

Instituto de Investigación en Educación

Universidad de Costa Rica

ISSN 1409-4703

http://revista.inie.ucr.ac.cr

COSTA RICA

\title{
LA ESTRATEGIA DE GRUPOS HETEROGÉNEOS EN EL NIVEL DE EDUCACIÓN INICIAL IMPLEMENTADA POR EL MINISTERIO DE EDUCACIÓN PÚBLICA DE COSTA RICA
}

STRATEGY OF HETEROGENEOUS GROUPS IN PREESCHOOL EDUCATION LEVEL IMPLEMENTED BY THE EDUCATION MINISTRY OF COSTA RICA

Volumen 9, Número 1

pp. 1-29

Este número se publicó el 30 de abril 2009

Cindy Álvarez Torres

Yency Araya Cantillano

Diana Herrera Solís

Karla Murillo Quirós

La revista está indexada en los directorios:

LATINDEX $, \underline{\text { REDALYC}}, \underline{\text { IRESIE}}, \underline{\text { CLASE}}, \underline{\text { DIALNET, DOAJ, E-REVIST@S}}$

La revista está incluida en los sitios:

$\underline{\text { REDIE}}, \underline{\text { RINACE}}, \underline{\text { OEI }}, \underline{\text { MAESTROTECA }} \underline{\text { PREAL }}, \underline{\text { HUASCARAN }}, \underline{\text { CLASCO }}$ 


\title{
LA ESTRATEGIA DE GRUPOS HETEROGÉNEOS EN EL NIVEL DE EDUCACIÓN INICIAL IMPLEMENTADA POR EL MINISTERIO DE EDUCACIÓN PÚBLICA DE COSTA RICA \\ STRATEGY OF HETEROGENEOUS GROUPS IN PREESCHOOL EDUCATION LEVEL IMPLEMENTED BY THE EDUCATION MINISTRY OF COSTA RICA
}

\author{
Cindy Álvarez Torres ${ }^{1}$ \\ Yency Araya Cantillano ${ }^{2}$ \\ Diana Herrera Solís ${ }^{3}$ \\ Karla Murillo Quirós ${ }^{4}$
}

\begin{abstract}
Resumen: En este artículo se analiza, mediante un estudio cuantitativo de tipo descriptivo, cómo se desarrolla la Estrategia Grupos Heterogéneos implementada por el Ministerio de Educación Pública en Escuelas Unidocentes y Dirección 1. Esta estrategia surge en el contexto nacional, en el año 2001, como un plan piloto denominado: Plan de Implementación del Programa de Estudio para el Ciclo Materno Infantil, a fin de responder a la situación de baja matrícula en algunas zonas rurales dispersas del país. De este modo, se conforman grupos de niñas y niños con edades entre los 4.3 años a los 6.6 años, quienes comparten el mismo espacio de trabajo y desarrollan las actividades en conjunto. Esta estrategia es relativamente nueva, por lo que surge la necesidad de analizar las implicaciones que su aplicación tiene para la realidad educativa nacional en lo que respecta a los actores del proceso educativo implicados en esta estrategia. Para esto, se aplicó un cuestionario tomando en cuenta variables como las características académicas y personales de las docentes, cantidad y edades de las niñas y los niños por grupo, funcionalidad del programa de estudio recomendado por el MEP, labor que realiza el personal con la familia y la comunidad, necesidades del personal en cuanto a formación continua y principales beneficios y limitaciones del trabajo con agrupaciones heterogéneas. Finalmente se elaboraron conclusiones en respuesta a dichas variables y recomendaciones dirigidas al Ministerio de Educación Pública, a las universidades y a las docentes que atienden estos grupos. En general se concluye que, la Estrategia de Grupos Heterogeneos tiene multiples ventajas tanto para los docentes que la aplica, como para la población estudiantil beneficiada; sin embargo, es necesario que entidades como el Ministerio de Educación Pública y Universidades que imparten la carrera de Educación Preescolar proporcionen las herramientas teorico-prácticas a las docentes que asumen estos grupos, siendo esta la principal debilidad manifestada por las entrevistadas.
\end{abstract}

Palabras claves: EDUCACIÓN INICIAL, ESTRATEGIA DE GRUPOS HETEROGÉNEOS, MINISTERIO DE EDUCACIÓN PÚBLICA, EDUCACIÓN RURAL, DESARROLLO INTEGRAL, COSTA RICA

\footnotetext{
Licenciada de Honor en Educación Preescolar de la Universidad de Costa Rica. Docente de Educación Inicial en el Colegio Saint Benedict. Dirección electrónica: at.cindy@gmail.com

2 Licenciada de Honor en Educación Preescolar de la Universidad de Costa Rica. Docente de Educación Inicial en Color Minds Montessori. Dirección electrónica: yencyac@gmail.com

${ }^{3}$ Licenciada de Honor en Educación Preescolar de la Universidad de Costa Rica. Dirección electrónica: dianaherrera18@hotmail.com

${ }^{4}$ Licenciada en Educación Preescolar, de la Universidad de Costa Rica.

Lugar de trabajo: Agencia de Aduanas Aeromar S.A.

Correo electrónico: karmuril@gmail.com
}

Artículo recibido: 8 de enero, 2009

Aprobado: 27 de abril, 2009 
Abstract: In this article it is analized, throught a quantitive descriptive study, how the strategy of Heteregoneus Groups is developed. The strategy was implemented by the Public Education Ministry and by schools classified as: Direction 1 and, "Unidocentes". The strategy comes up based on a national need, in 2001. It began as a Pilot Plan, denomitated: "Implementation Plan for the Nursery Level Program". Basically, it was created in order to respond to the situation of low enrolment, at this level, in some scattered countryside areas in Costa Rica. In this way, groups of children in ages between the 4,3 years to the 6,6 years share the same working and learning space, and develop the same activities altogether. This strategy is relatively new, which means that analyzing its implications becomes necesary, regarding all of those involved in the educational process.

For this, a questionnaire was applied taking into account variable like the academic and personal characteristics from the teachers, amount and ages of the children by group, functionality of the training program recommended by the MEP, work that realises the personnel with the family and the community, needs of the personnel as far as continuous formation and main benefits and limitations of the work with heterogenous groupings. Finally we made conclusions in response to these variables and recommendations directed to the Ministry of Public Education, the universities and the teachers of these groups. Generally one concludes that, the Strategy of Heterogenous Groups as much has multiple advantages for the teachers that works with this, and for the children; neverthdess is necessary that organizations like the Ministry of Public Education and Universities that teachs the race of Preschool Education provide to the teachers theoretical-practices tools, being this the main weakness indicated by the interviewed ones.

Keywords: INITIAL EDUCATION, HETEROGENOUS GROUPS STRATEGY, PUBLIC EDUCATION MINISTRY, RURAL EDUCATION, INTEGRAL DEVELOPMENT, COSTA RICA

\section{Introducción}

El Ministerio de Educación Pública (MEP) siempre ha seguido la tendencia de organizar los grupos de niñas y niños de forma homogénea en el nivel de educación inicial, sin embargo, en los últimos años se han originado diversos cambios en el contexto social y económico que dan como consecuencia una transformación de esta modalidad. Uno de estos cambios es la situación de baja matrícula que se presenta en algunas zonas rurales dispersas del país; esto ha generado la aparición de las agrupaciones heterogéneas, verticales o familiares que se caracterizan "por la integración en un mismo grupo de niñas y niños con edades cronológicas, niveles de desarrollo, intereses y necesidades diferentes" (MEP, 2004, p. 85).

En el año 2001 en el Departamento de Educación Preescolar del MEP surge la idea de llevar a cabo un plan piloto denominado "Plan de Implementación del Programa de Estudio para el Ciclo Materno Infantil" con el fin de ampliar la cobertura de la Educación Inicial y garantizar la equidad en el acceso a la educación por medio del uso de ese programa de estudio en Escuelas Unidocentes y Dirección 1. Es así como surge la llamada Estrategia de Grupos Heterogéneos ${ }^{5}$ en la que se empiezan organizar, en un mismo grupo, a niñas y niños

\footnotetext{
${ }^{5}$ Trabajo Final de Graduación (TFG) dirigido por la Dra. Lupita Chaves Salas, para optar por el grado de Licenciatura en Educación Preescolar, Escuela de Formación Docente. Universidad de Costa Rica. 
tanto del Grupo Interactivo II (4.3 años a 5.6 años) como del Grupo de Transición (5.3 años a 6.6 años). En un inicio, esta estrategia era aplicada en 21 códigos educativos y se había ampliado de modo tal que para el año 2007 ya existían 996 códigos en 20 regiones educativas del país.

Tomando en cuenta que esta es una estrategia de aplicación reciente en nuestro país, y que la mayor parte de los estudios relacionados con esta temática son de carácter extranjero, surgió la idea de realizar una investigación en torno a este tema que respondiera a la siguiente interrogante:

¿Cómo se desarrolla la Estrategia de Grupos Heterogéneos en el nivel de Educación Inicial desarrollado por el Ministerio de Educación Pública en las Escuelas Unidocentes y Dirección 1 ?

A partir de esta interrogante surge un objetivo general y seis específicos, a saber:

\section{Objetivo General}

Analizar la Estrategia de Grupos Heterogéneos en el nivel de educación inicial que está desarrollando el Ministerio de Educación Pública en las escuelas Unidocentes y Dirección

\section{Objetiv os Específicos}

1. Identificar las características académicas y personales de las y los docentes que atienden a las niñas y los niños en la Estrategia de Grupos Heterogéneos en el nivel de Educación Inicial.

2. Determinar la cantidad y las edades de las niñas y los niños por grupo que se atiende en la Estrategia de Grupos Heterogéneos

3. Determinar la funcionalidad del programa de estudio recomendado por el Ministerio de Educación Pública para el trabajo con la Estrategia de Grupos Heterogéneos.

4. Identificar la labor que realizan las educadoras con las familias y la comunidad.

5. Determinar las necesidades en cuanto a formación continua de las educadoras que laboran en esta estrategia.

6. Determinar los principales beneficios y limitaciones del trabajo con agrupaciones heterogéneas en el contexto costarricense. 


\section{Referente Teórico}

\subsection{Importancia de la Educación Inicial}

Proveer de un ambiente material, espacial y humano adecuado para la formación holística de las niñas y los niños ha sido una de las principales causas que ha incentivado al ser humano a proponer e implementar sistemas que cubran las necesidades de formación integral de la futura ciudadanía de sus sociedades; estos sistemas deben responder a las expectativas de vida de los pueblos, sus costumbres y otras necesidades socioeconómicas relativas al momento histórico en que se está viviendo.

La Educación Inicial, como acto intencional, viene gestándose desde el advenimiento de la Revolución Industrial, cuando surge la necesidad de crear centros de atención infantil para suplir las necesidades de las madres, quienes comienzan a insertarse en el mercado laboral y necesitan una alternativa segura para el cuido de sus hijos (Zúñiga, 2001, p. 19).

Al pasar el tiempo, brindar una mejor educación que potencie el desarrollo de la niña y el niño se volvió el objetivo principal de la Educación Inicial, muchos autores concuerdan con que el ser humano debe ser formado durante sus primeros años de vida, y exponen la importancia de la individualidad de la niña y el niño además de su concepción específica del desarrollo particular. Formalmente, Federico Froebel fue el primero que logró consolidar una propuesta teórica y práctica para la atención de las niñas y los niños preescolares, proponiendo el desarrollo como un proceso continuo y progresivo, donde "cada etapa requiere que se le atienda plenamente, en sí misma y en función del desarrollo global (Zuñiga, 2001, p. 21).

En Costa Rica, las primeras ideas de incluir un componente pedagógico al trabajo en los jardines infantiles fueron las del modelo introducido en 1878 , que era de corte meramente asistencial y religioso, las llamadas Escuelas para Párvulos (Zúñiga, 2001, p. 19). Fue hasta 1926 cuando María Isabel Carvajal (Carmen Lyra) funda, junto a Luisa González y Margarita Castro, la Escuela Maternal Montessoriana, primer jardín infantil con orientación pedagógica de nuestro país. Con ellas se dan los primeros pasos para estructurar el Nivel Inicial de tal manera que hoy en día es considerado uno de los niveles más importantes de la educación formal, incluso podría decirse que el más esencial ya que abarca los primeros años de vida del individuo. Pues, como lo mencionan Arenas, Castro, Gómez, Hernández, Méndez, Núñez, Sánchez, Reyes, Santiago y Rivas (2007, p. 4) la etapa de la primera infancia

...es considerada en general como la más significativa del individuo, debido a que en esta se estructuran las bases fundamentales de las particularidades físicas y Volumen 9, Número 1, Año 2009, ISSN 1409-4703 
psicológicas de la personalidad, así como de la conducta social que en las sucesivas etapas del desarrollo se consolidarán y perfeccionarán.

Por este motivo, es importante que la Educación Inicial se centre en brindar al sujeto las herramientas necesarias que le permitan desarrollarse de manera integral e incorporarse con éxito en la sociedad, para lo que es necesario una adecuada estimulación y nutrición desde edades tempranas, aprovechando así las condiciones que a nivel de desarrollo están presentes en esta etapa de la vida.

La Educación Inicial se ha ganado un lugar destacado en el Sistema Educativo y actualmente se constituye como un nivel primordial debido a que atiende la etapa base del desarrollo en donde se dan la mayor parte de los procesos evolutivos de una manera acelerada y es el momento en que la niña y el niño tienen mayor facilidad para incorporar nuevos aprendizajes. Al respecto,

Es por ello, que la atención integral en estas edades debe visualizarse no sólo desde una perspectiva asistencial, sino también con un fuerte componente pedagógico, que involucra las diferentes áreas del desarrollo infantil, los derechos de las niñas y los niños, la satisfacción de necesidades básicas, así como el respeto por el contexto social, cultural e histórico de pertenencia. (Blanco y otros, 2002, p. 5)

Hoy en día tiene un gran auge la tendencia a adoptar una estrategia de agrupación en la que se mezclan diversas edades, es decir, agrupaciones heterogéneas. Esta estrategia supone múltiples beneficios en todas las áreas del desarrollo, además de los ya reconocidos para el Nivel Inicial.

Según el MEP (2004, p. 85), el propósito de estas agrupaciones es "favorecer una integración similar a la de una familia, donde se compartan experiencias e intereses con personas de diferentes edades".

A pesar de que, en nuestro país este tipo de agrupación se ha venido implementando en muchos centros infantiles de carácter privado y algunos de carácter público, es hasta el año 2001 cuando el MEP lo integra como estrategia para el trabajo en el Nivel Inicial, con la finalidad de garantizar el acceso a la Educación Preescolar de las niñas y los niños pertenecientes a zonas rurales dispersas, donde la matrícula es muy baja, respaldando con esto los principios de equidad e igualdad de oportunidades de la población infantil costarricense (Blanco y otros, 2002). 
El trabajo con la estrategia heterogénea puede presentar serias dificultades, sin embargo, existen evidencias de que son muchos los beneficios que esta trae para el desarrollo integral de las niñas y los niños y para la población docente que la pone en práctica. En un grupo heterogéneo es normal que las niñas y los niños con aprendizajes más avanzados ayuden y expliquen nuevos conceptos a los de menor edad, quienes -sin dudase ven favorecidos, esto nos lleva a la idea de aprendizaje asistido propuesto por Vigotsky, el cual se da a partir de interacciones con personas más conocedoras que brindan el apoyo requerido para lograr nuevas habilidades y conocimientos, este apoyo es conocido como andamiaje (Papalia, Wendkos y Duskin, 2001).

El concepto de andamiaje resulta de gran validez en la Estrategia de Grupos Heterogéneos, pues el apoyo que reciben las niñas y los niños de menor edad les da la oportunidad de adquirir nuevos conocimientos y habilidades en las diferentes áreas que los harán pasar a un nivel superior de desarrollo; del mismo modo, los más avanzados tendrán la posibilidad de reafirmar sus conocimientos y su autoestima al saberse útiles para los demás. Además, las niñas y los niños mayores demuestran mayor capacidad de liderazgo y manifiestan actitudes de sensibilidad y cooperación.

Del mismo modo, según Katz (2005), las niñas y los niños de mayor edad logran controlar y mejorar su conducta cuando son estimulados para que ayuden a los más pequeños a cumplir las reglas de la clase. También, las niñas y los niños menos maduros socialmente del grupo se ven beneficiados con esta estrategia, pues a menudo son más aceptados por los más pequeños en el grupo de diferentes edades que por sus pares.

Por otra parte, las niñas y los niños menores, al ser ayudados por los de mayor edad, logran aprender modelos positivos de conductas sociales caracterizados por la cooperación, la solidaridad y la empatía que, posiblemente, pondrán en práctica cuando ellos sean los mayores.

Aunado a esto, el hecho de que las niñas y los niños se desenvuelvan en una agrupación heterogénea los hace ver que cada uno es una persona única, lo que, según Sundell (1993), promueve la formación de seres cada vez más tolerantes y abiertos de mente, además de que contrarresta la competencia.

En cuanto a la labor docente, Sundell (1993) menciona que este tipo de agrupación tiende a influir negativamente en la manera de enseñar de la maestra o maestro; destaca que en grupos donde hay gran brecha de edad entre los niños más pequeños y los mayores, 
hay pocas actividades dirigidas. Según el mismo autor, esto puede provocar que las niñas y los niños cuenten con menos competencia cognitiva.

Los primeros seis años de vida son vitales para el desarrollo de la persona y por este motivo, los estímulos que reciba del medio van a garantizar la potencialización o no de dichas capacidades, de manera que es necesaria una intervención adecuada durante estos años, que idealmente debe desarrollarse desde una visión holística partiendo de las características y necesidades individuales de la persona.

Con respecto a las ventajas y limitaciones que diferentes autores señalan propias de este tipo de estrategia, el equipo investigador adopta una posición que apoya la idea de que los beneficios obtenidos del trabajo con grupos heterogéneos se ven reflejados en todas las áreas del desarrollo y son diferentes de los que se obtienen con una agrupación homogénea. En el área socioafectiva, las niñas y los niños tienen la posibilidad de interactuar con otros que, al ser mayores o menores que ellos, les darán la oportunidad de aprender e incluso enseñar y desarrollar valores de respeto, cooperación, empatía y afectividad.

No obstante, se considera que el progreso en las otras áreas del desarrollo se logrará de acuerdo con el abordaje que le dé el equipo docente a la práctica pedagógica con una agrupación heterogénea.

\subsection{El currículo en la Educación Inicial}

La Educación Inicial debe de ser una actividad pensada y planificada, por tanto, debe exigir del personal docente y de la institución educativa la realización de una tarea comprometida que contenga en sí misma una serie de argumentos y bases teóricas para su total eficacia, esto debido a que las necesidades del mundo actual son numerosas y los retos del profesorado son cada vez más complejos, estos hechos implican una tremenda responsabilidad moral y profesional en la labor docente.

Es necesario definir lo que se considera como currículo, que es un todo complejo y organizado, que contempla el qué, el por qué y el para qué enseñar. Esto se logra al incluir una serie de elementos como los programas de estudio, la programación o planificación y el proceso de enseñanza en sí.

De este modo, la educación de cada país difiere, pues adopta una concepción de currículo propia y que se adapte a su contexto. En el caso de Costa Rica, es el Ministerio de Educación Pública el que se encarga de elaborar y reestructurar el currículo oficial, basándose en diversos postulados teóricos. 
Peralta (1988, p. 6) presenta un referente conceptual de definiciones y postulados teóricos, donde determina inicialmente al currículo como

Todas las vivencias y aprendizajes significativos que han sido experienciados por los párvulos y adultos, como resultante de la selección y organización consistente de un conjunto de factores humanos, materiales y técnicos, que han sido generados por una comunidad educativa, desde el Jardín Infantil como institución educativa sistémica

Ahora bien, se presenta la necesidad de hacer alusión a los planteamientos curriculares relacionados con grupos heterogéneos. Uno de los primeros teóricos, que en su propuesta curricular contempla la mezcla de edades, fue Frédéric Froebel, quien enfoca su labor bajo un criterio de agrupación mixta conformada por niñas y niños de uno a seis años. Sin embargo, fue María Montessori quien desarrolló un método donde trabajaba con grupos heterogéneos específicamente. Estas agrupaciones eran llamadas grupos familiares y estaban constituidas por niñas y niños de tres a seis años (Peralta, 1988).

A nivel nacional se ha implementado el Programa de Estudio para el Ciclo Materno Infantil como una guía para la programación o planificación curricular en las agrupaciones heterogéneas; además, existe la opción de utilizar el Programa para el Ciclo de Transición, pero solamente para el empleo de los procedimientos que plantea. Para el MEP, dentro de los fundamentos básicos de esta propuesta curricular se encuentran el filosófico, el psicológico, el social-cultural, el ecológico, el biológico y el pedagógico.

De acuerdo con Licda. Elizabeth Montero Campos, Asesora Nacional de Educación Preescolar, la planificación para grupos heterogéneos debe ser para un lapso entre 8 y 15 días, dependiendo del tema que se aborde y del interés real del grupo de niñas y niños. La manera en que se lleva a cabo la planificación curricular en el Nivel Inicial, según lo establece el Programa de Estudios para Ciclo Materno Infantil, es mediante la utilización de propósitos, que serían similares a los objetivos, los cuales se estructuran tomando como base cuatro "núcleos generadores" que toman en cuenta las siguientes áreas : sociocultural, personal, de conocimientos y de comunicación. (MEP, 2004)

Cabe destacar que la planificación en la Educación Inicial no solamente toma en cuenta el trabajo con el grupo de niñas y niños, sino que incluye el desarrollo de actividades con la comunidad y las familias, de modo que se teje un sistema de interacciones entrelazadas con el fin de dotar a la niña y al niño con la mayor cantidad de estímulos posibles. 
Para el trabajo con familia en la Estrategia de Grupos Heterogéneos se sugiere la utilización del programa Ventanas en el Mundo Infantil. Este programa, gestado por el Departamento de Educación Preescolar del Ministerio de Educación, busca "privilegiar una cultura institucional más fresca y centrada en que cada quien asuma sus responsabilidades".... (MEP, 2007, p. 3). Para ello, propone un proceso de interaprendizaje, entendido como "una vivencia personal y colectiva que favorece la comunicación e interacción entre las personas que participan, así como la construcción de conocimientos por medio del intercambio de experiencias, es decir, aprender entre todas las personas" (MEP, 2007 , p. 15); en esta experiencia se sugieren como participantes al personal educativo, la comunidad y la familia de las niñas y niños, pues esta última es considerada de gran trascendencia para el desarrollo integral de las y los estudiantes.

Para este programa se editaron y publicaron siete fascículos para la familia y la comunidad que abordaban diferentes temáticas; un material radiofónico, comprendido por cuarenta programas de audiencia cerrada -utilizados por el cuerpo docente en los talleres de interaprendizaje- y cuarenta programas de audiencia abierta -programas radiofónicos transmitidos en las radioemisoras culturales de todo el país - y, por último, encontramos el fascículo para la mediación, formulado para convertirse en una herramienta con la propuesta didáctica y metodológica para el abordaje educativo de la serie Ventanas en el Mundo Infantil.

Por otra parte, con respecto a la evaluación, en Preescolar el fin no es medir conocimientos, sino llevar un control del desarrollo de cada niña y cada niño, de sus necesidades e intereses y cómo éstos están siendo subsanados, es decir, saber qué acciones son pertinentes y cuáles no lo son en la práctica docente.

Esta evaluación se establece dentro de un enfoque cualitativo y debe darse a través de la observación, por ende, utiliza instrumentos que permiten -más que dar una nota- evaluar el progreso de la niña y del niño, como por ejemplo: listas de cotejo, registros descriptivos y anecdóticos, evaluación de desempeño, escalas de valoración, entre otros.

Además, no sólo la planificación cumple un papel determinante en el proceso educativo, "(...) la organización espacial y temporal de las experiencias, así como los materiales, la planificación e interacciones con el contexto, cobran especial importancia para el desarrollo integral de los sujetos involucrados en la acción educativa"' (MEP, 2004, p. 48). Por lo tanto, se propone que éste sea rico en estímulos y que a nivel de aula se organice por espacios de interacción, los cuales se conocen como: espacio para el recibimiento, para el Volumen 9, Número 1, Año 2009, ISSN 1409-4703 
sueño o descanso para la alimentación, para la higiene y aseo personal, para archivo y exposición, para el juego, para el movimiento, para juegos que desarrollan la actividad perceptiva, para la comunicación y expresión, para la identificación y la representación, para la cría de animales y el cultivo de plantas (MEP, 2004). Los mismos se utilizan durante la rutina diaria de manera flexible y sin dejar de lado los intereses y necesidades de las niñas y los niños.

También, la duración de las actividades que se desarrollararán es muy importante en el proceso educativo. En este sentido, las actividades deben tener una secuencia lógica y respetar los tiempos establecidos por las niñas y los niños. El MEP (2004) propone las llamadas Experiencias de Libre Acción, las Estructuradas e Intermedias; de modo que la docente pueda jugar con estos tipos de actividades y disponga del tiempo necesario para cada una.

Por otra parte, existe la interacción con los materiales, estos vienen a ser otro de los medios de construcción de conocimientos empleados por las niñas y los niños. En este sentido, se considera importante su ubicación, durabilidad, funcionalidad, objetivos que se pretenda alcanzar con su uso, calidad, composición, etc.

\subsection{Formación de docentes en Educación Inicial}

Debido a que la acción educativa se encuentra, en mayor medida, en manos del personal docente este debe contar con ciertas cualidades personales, además de profesionales que lo faculten para desempeñar una práctica responsable, conciente y pertinente a la población con la que trabaja.

Una de las cualidades personales con la que debería contar cualquier docente es la "vocación". Al respecto, Arguedas, Bustos, Chinchilla, González, Hutchinson y Vargas mencionan lo siguiente

En primer término el docente debe de poseer vocación porque será la que genere la motivación en la labor que desempeñe; además las personas que eligen esta profesión lo hacen por la trascendencia que tiene en la sociedad, más que por un sentido lucrativo. (1999, p. 42)

De manera más específica, en el campo de la Educación Inicial, el personal docente debe estar preparado para realizar un trabajo enfocado no solamente en el desarrollo cognitivo, como es la tendencia en niveles superiores, sino que también debe contemplar el desarrollo de la niña y el niño de manera integral. De este modo, se exige que cuente con habilidades personales y profesionales que le permitan interactuar con los niños y las niñas Volumen 9, Número 1, Año 2009, ISSN 1409-4703 
ya que "la (el) docente de Educación Inicial (Preescolar) está permanentemente relacionada(o) con niños(as) y los acompañan en la construcción de sus pensamientos, sentimientos y en sí de toda su personalidad' (Bolaños, Cortes, Maroto, Rojas, Solano y Villalobos, 2004, p. 189).

Todo docente debe tratar de funcionar como mediador del proceso de enseñanza aprendizaje, que permita un ambiente en el que las niñas y los niños desarrollen habilidades de acuerdo con sus necesidades e intereses y tengan la posibilidad de construir su conocimiento y de "aprender a aprender", lo que significa que sean capaces de elaborar esquemas mentales al entrar en contacto con su medio.

Ahora bien, tomando en cuenta las necesidades de la sociedad actual, los constantes avances y las nuevas exigencias en el ámbito educativo y social las universidades, tanto estatales como privadas, han creado sus planes de estudio para la formación de profesionales en el área de la Educación Inicial, estos tienen "el desafío de responder oportuna y adecuadamente a los requerimientos de esta sociedad en cuanto al desarrollo integral de los niños y niñas del presente" (Picado, 2007, p. XI).

Las transformaciones que se han dado en la sociedad actual han generado la necesidad de darle un giro a la manera en que se llevan a cabo los procesos pedagógicos. Uno de estos cambios ha sido el surgimiento de diferentes estrategias y contextos en los que se puede llevar a cabo la labor docente, como es el caso de las agrupaciones heterogéneas en el Nivel Inicial conformadas en el ámbito educativo costarricense durante la última década. Ante esta situación surge la necesidad de que la formación de los profesionales en educación se adapte a las demandas de este tipo de agrupaciones que no sólo difieren en la edad de las niñas y los niños, sino también en la manera en que el personal docente debe desarrollar su labor.

Además de las características básicas con las que debe contar todo profesional en Educación Inicial, quienes trabajen con este tipo de agrupaciones deben poseer ciertas características adicionales que les permitan desarrollar una práctica que resulte pertinente y sea satisfactoria para todos los implicados en el proceso.

De esta manera, las universidades deben diseñar planes de estudio que garanticen innovaciones en cuanto a los procesos formativos y, así, responder a este tipo de necesidades, permitiendo al personal docente desempeñarse de manera óptima con cualquier tipo de agrupación con la que tenga la oportunidad de trabajar.

Aparte de la formación básica provista por las universidades, todo profesional en educación debe procurar una formación continúa que le permita actualizarse en cuanto a las Volumen 9, Número 1, Año 2009, ISSN 1409-4703 
nuevas tendencias y estudios aplicados en el campo de su competencia. Al respecto, Berrocal, Campos, Sáenz y Torres (1993, p. 39) señalan que "Los maestros titulados en educación deben continuar capacitándose por sí mismos, enriqueciendo sus conocimientos con los nuevos avances que se dan en el campo educativo, para actuar acorde a la realidad y al desarrollo de la sociedad".

Dichas actualizaciones no necesariamente se dan a través de la participación en programas, capacitaciones o cursos que impartan diversas entidades, sino que además es válido recurrir a la búsqueda de información en diferentes fuentes bibliográficas. No obstante, se consideran de vital importancia los programas de capacitación impartidos por expertos, que atiendan las necesidades y los vacíos en cuanto a formación que el personal docente manifiesta.

Para Lafourcade, citado en Berrocal y otros (1993, p. 52), "es importante conocer la opinión de los egresados respecto a los conocimientos que deben poseer y las tareas que deben desempeñar en función del bienestar social".

La situación social y económica actual así como los cambios en la matrícula de los centros públicos han generado la necesidad de que el cuerpo docente esté preparado para retos cada vez más complejos y para los cuales, en muchos de los casos, no cuentan con la orientación ni la formación adecuada que los faculte para asumir estos desafíos contemporáneos, por ello, es necesario que el profesorado sea inquisitivo y crítico en su labor y mantenga como una constante el mejoramiento y la formación. Al respecto, García (1999, p.7) menciona

(...) resulta necesario favorecer la formación, la situación social y las condiciones de trabajo de los docentes, pues difícilmente podrán responder a las nuevas exigencias sino poseen los conocimientos y la competencia, las cualidades personales y la suficiente motivación hacia la tarea profesional.

Para poder responder a las demandas de la sociedad, el cuerpo docente, desde una perspectiva sociológica, es un agente de transformación social y sus responsabilidades trascienden el ámbito del aula, exigiendo de él cada vez más creatividad, innovación, flexibilidad y un amplio sentido de pertinencia que lo inste a no cesar en la búsqueda del mejoramiento de su labor docente.

Se concluye que, para desarrollar una práctica educativa de calidad, cada docente -en especial cuando trabaja con agrupaciones heterogéneas- debe contar con ciertas Volumen 9, Número 1, Año 2009, ISSN 1409-4703 
características dentro de las que se destacan: un amplio conocimiento acerca del desarrollo, la pedagogía y currículo infantil; flexibilidad, innovación, capacidad para la resolución de problemas y la adaptación al cambio, de observación y escucha, creatividad, tolerancia y respeto hacia la individualidad, estabilidad emocional, asertividad, alto compromiso social, empatía, criticidad consigo mismo y su entorno, reflexivo, entusiasta, investigador, emprendedor, conocedor y transmisor de los valores, la cultura y el arte, idealista y con una visión perseverante que le permita comprometerse con la transformación de la realidad, en vez de conformarse con lo que ya está establecido.

\section{Metodología}

Se llevó a cabo un estudio cuantitativo de tipo descriptivo; para desarrollarlo se dividió la metodología en seis etapas:

\section{Etapa. Indagación documental:}

Durante esta etapa se realizó una búsqueda bibliográfica en diferentes bases de datos, en Internet y en el Sistema de Bibliotecas, Documentación e Información de la UCR (SIBDI); además, se realizaron entrevistas a la Licda. Elizabeth Montero, asesora nacional del Departamento de Educación Preescolar del MEP. La información recolectada durante esta etapa sirvió como base para la elaboración del marco teórico y los antecedentes históricos del presente trabajo.

Además, se realizaron entrevistas a las coordinadoras de la carrera de Educación Inicial de la Universidad de Costa Rica, de la Universidad Nacional, de la Universidad Estatal a Distancia y de la Universidad De La Salle; con estas se buscó indagar acerca del tipo de formación que reciben las docentes de Educación Inicial.

\section{Etapa. Elaboración y validación de instrumentos:}

Tomando en cuenta los objetivos de la investigación, se diseñó un cuestionario dirigido a docentes que trabajan con la Estrategia de Grupos Heterogéneos. Este instrumento tenía como finalidad conocer cómo desarrollan su práctica educativa las docentes que laboran en este tipo de agrupación y algunos aspectos relevantes acerca de su experiencia en esta estrategia.

Para validar el instrumento, se realizó un juicio de expertos a cargo de la M.Sc. Ana Isabel Cerdas (Directora del Departamento de Educación Preescolar del MEP), la Licda. Volumen 9, Número 1, Año 2009, ISSN 1409-4703 
Elizabeth Montero (Asesora nacional del MEP) y la Licda. Magally Gutiérrez (Profesora del Departamento de Educación Inicial de la UCR). Asimismo, se aplicó el cuestionario, con fines de validación, a siete maestras que trabajaban aplicando la estrategia en las regiones educativas de Heredia y San José.

III Etapa. Definición de la población y la muestra:

La población consistió en la totalidad de docentes que trabajan aplicando la Estrategia de Grupos Heterogéneos; para el año 2007 estaba conformada por 996 docentes ubicadas en 20 regiones educativas del país.

La muestra fue elegida al azar y estuvo conformada por ciento veintiún docentes pertenecientes a catorce regiones educativas. En su mayoría eran docentes de las regiones de Pérez Zeledón y Nicoya.

\section{Etapa. Aplicación de instrumentos.}

Para la aplicación de los instrumentos se contactó a las asesoras de las diferentes regiones educativas del país, de este modo se recolectaron diecisiete instrumentos de la región de San Carlos, veinticuatro instrumentos en Pérez Zeledón, seis instrumentos en Cartago, trece instrumentos en Santa Cruz, nueve instrumentos en Liberia, dieciecho instrumentos en Cañas y veintitrés instrumentos en Nicoya. Además se asistió a una capacitación en la que se lograron recolectar tres instrumentos de la region de Upala, tres de Coto, dos de San Ramón, uno de Aguirre, uno de Guápiles y uno sin el dato de la región educativa. Al concluir esta etapa se lograron recolectar ciento veintún instrumentos.

\section{Etapa. Análisis de la información recolectada}

Para el análisis de la información recolectada por medio de los cuestionarios se tuvieron en cuenta seis variables y sus respectivos indicadores (ver anexo 1).

\section{Etapa. Elaboración de conclusiones y recomendaciones}

Tomando en cuenta los resultados de la investigación, se llegó a varias conclusiones y se realizaron recomendaciones con el fin de enriquecer y mejorar esta estrategia. Las conclusiones se elaboraron tomando en cuenta los objetivos planteados y tratando de responder a las interrogantes planteadas en ellos. Las recomendaciones fueron dirigidas a 
tres entes principales: el MEP, las universidades que forman profesionales en Educación Inicial y el personal docente que labora con la Estrategia de Grupos Heterogéneos.

\section{Análisis de la información}

El análisis de la información recolectada se realizó de acuerdo con seis variables establecidas y sus respectivos indicadores, los cuales responden a los objetivos específicos del presente trabajo. Las variables son las siguientes:

\subsection{Características sociodemográficas de la muestra}

Con respecto a las características personales y profesionales de las docentes que trabajan con la Estrategia de Grupos Heterogéneos, los resultados muestran que la totalidad fue conformada por mujeres, en su mayoría en edades entre los 25 y 34 años (57.02\%), con un grado académico de Licenciatura (77.69\%) y egresadas de la Universidad Latina (42.97\%).

La experiencia profesional en el campo de la Educación Inicial de las educadoras va de 1 a 10 años (96.09\%) y específicamente en el trabajo en la Estrategia de Grupos Heterogéneos la experiencia es de 1 a 3 años (88\%).

En cuanto a la formación continua, el estudio mostró que, de las docentes de la muestra, solamente el $28.10 \%$ continúa estudiando. Este hecho es destacable, pues la actualización y la búsqueda de nuevos conocimientos debe ser parte de las labores que todo profesional en educación debe desempeñar. Como lo señalan Berrocal y otros (1993, p.39), los profesionales en educación "deben continuar capacitándose por sí mismos, enriqueciendo sus conocimientos con los nuevos avances que se dan en el campo educativo, para actuar acorde a la realidad y al desarrollo de la sociedad".

Para estos fines, las coordinadoras de la carrera de Educación Preescolar de universidades como la UCR, la UNA, La Salle y la UNED reconocen la importancia de desarrollar actividades (simposios, cursos, talleres, congresos, etc.) con enfoque hacia la comunidad y el profesorado con fines de capacitación y formación de estos profesionales. 


\subsection{Desarrollo didáctico y metodológico}

\section{Organización de los grupos}

En lo referente a la conformación de los grupos, los resultados mostraron que el $80.98 \%$ estaba formado por un mínimo de 6 y un máximo de 15 niñas y niños con edades entre los 4.3 y 6.6 años, esto responde de manera coherente con los planteamientos establecidos por el MEP.

Es importante que la cantidad de niñas y niños que conforman cada grupo no exceda los 15 estudiantes, pues esto permite desarrollar una labor de atención personalizada y facilita la planificación y control de grupo de las docentes que trabajan con esta estrategia.

La jornada laboral que cumple el $86.77 \%$ de las educadoras es de 4 horas y 10 minutos, la cual es la establecida por el MEP para el trabajo con estas agrupaciones.

\section{La Planificación}

La planificación didáctica "otorga a la experiencia pedagógica racionalidad, intencionalidad y coherencia" (MEP, 2004, p.106). Esta se encuentra conformada por tres elementos que se interrelacionan entre sí, uno de estos es el planeamiento didáctico.

Según la información brindada por las docentes sólo $63.63 \%$ de ellas planea según el lapso propuesto (8 a 15 días). Sin embargo, un $30.58 \%$ de la muestra lo hace de manera mensual y, aunque lo dividen en dos quincenas, es importante cuestionar si este planeamiento es flexible y sigue realmente los intereses y las necesidades de las niñas y los niños, pues es evidente que en un mes estos pueden variar mucho y pueden volver obsoleto el plan a ejecutar.

Otro de los aspectos por conocer son las fuentes de las que surge este planeamiento didáctico; a pesar de que el MEP en sus programas de estudio da una serie de lineamientos y temáticas en las que este puede ser basado, es a partir de las características, intereses y necesidades de las niñas y los niños de donde debe surgir la planificación para ser, con certeza pertinente y significativa.

Según los datos obtenidos en la investigación, el 14.88\% de las docentes deja estos aspectos de lado, fundamentando su planeamiento únicamente en sus criterios o en los del MEP. Resulta meritorio rescatar que el $17.35 \%$ de las docentes, además de tomar en cuenta las características de las niñas y los niños, se basan en los intereses y las necesidades tanto de la familia como de la comunidad, quienes influyen de manera constante en el individuo y Volumen 9, Número 1, Año 2009, ISSN 1409-4703 
constituyen el entorno en donde se desarrolla, lo que favorece, de manera significativa, su desarrollo integral.

Ahora bien, a nivel teórico, para realizar el planeamiento didáctico según los lineamientos del MEP, se debe utilizar como guía el Programa de Estudio para el Ciclo Materno Infantil y el Programa de Transición para la extracción de procedimientos. Sin embargo, además de tomar en cuenta estos programas de estudio (que son unicamente una guía), queda en manos del profesorado indagar en otras fuentes para realizarlo. Se encontró que un $43.63 \%$ de las profesionales en Educación Inicial recurren a libros, enciclopedias, revistas, Internet, o profesionales en diversas áreas; este es un dato significativo dado que permite a la especialista enriquecerlo y hacerlo más significativo y atractivo para las niñas y los niños.

Otro elemento de gran valor que constituye la planificación didáctica es la evaluación, proceso que acompaña y complementa las tareas de diagnóstico y planeamiento que realiza la docente, a partir de la cual se toman decisiones respecto a los cambios o adaptaciones curriculares. (MEP, 2004, p.111). Se observa que los instrumentos de evaluación más utilizados por las docentes que trabajan con la Estrategia de Grupos Heterogéneos son el registro anecdótico, las entrevistas y la lista de cotejo. Este aspecto no es de extrañar debido a que para el trabajo con agrupaciones heterogéneas se recomienda la evaluación por observación de tipo cualitativo en cada momento del proceso educativo, lo que consideramos pertinente para la labor pedagógica que se desarrolla en los centros infantiles.

\section{Manera en que las docentes integran a la familia en el proceso educativo}

El fortalecimiento del vínculo escuela-familia es de gran importancia para favorecer el desarrollo de las niñas y los niños dado que estas entidades constituyen los principales contextos educativos en que ellos se desenvuelven y pueden convertirse en un gran apoyo para la labor pedagógica del docente.

De acuerdo con la información obtenida, se puede observar que las principales maneras en que las docentes incluyen a la familia en el proceso educativo son por medio de invitaciones a actividades en el aula (91.73\%), reuniones (75.21\%), e invitaciones a actividades extra-curriculares (67.77\%). En este punto es necesario destacar que el cuerpo docente debe preocuparse porque estas actividades sean de interés real para las familias, de modo que aseguren su participación en el proceso. 
Además, se hace hincapié en que sin más un $0,83 \%$ de las docentes utilizan las visitas al hogar como opción para integrar a las familias en el proceso educativo, haciendo notar que esto se ha ido perdiendo, lo que es lamentable, pues conocer la realidad que viven sus estudiantes y las interacciones que se dan en cada hogar permite al docente realizar una planificación pertinente que responda a las necesidades individuales de las niñas y los niños.

\section{Manera en que las docentes integran a la comunidad en el proceso educativo}

Otro vínculo importante por fortalecer es el de la escuela-comunidad, pues es uno de los entes socializadores que influyen de manera directa en las niñas y los niños. Una de las principales formas en que las docentes incluyen a la comunidad en el proceso educativo es por medio de actividades a beneficio a la institución, realizando acciones culturales con las niñas y los niños en la comunidad e invitando a especialistas a reuniones con la familia.

Es importante rescatar que la gran mayoría utiliza actividades a beneficio a la institución lo cual genera la duda de si de esta manera la comunidad participa en efecto de forma activa dentro del proceso, o bien, sólo es utilizada para recaudar fondos materiales y económicos, como es queja de muchas instituciones.

Necesidad de crear un programa de estudio específico para el trabajo con la Estrategia de Grupos Heterogéneos

Al saber que el programa recomendado para el trabajo con esta estrategia es el Programa de Estudio para el Ciclo Materno Infantil, surgió la necesidad de saber si las docentes consideran necesaria la creación de un programa de estudio específico para el trabajo con agrupaciones heterogéneas; el $84.30 \%$ de la muestra respondió que sí, mientras que el $12.40 \%$ respondió que no. La mayor parte de las docentes manifestó que un nuevo programa les permitiría desempeñarse mejor como profesionales.

Son las docentes que trabajan con esta estrategia quienes poseen el conocimiento empírico de las necesidades y los puntos débiles de la labor que desempeñan, por tal motivo, se les preguntó acerca de los aspectos que incluirían en un posible programa de estudio específico para el trabajo con esta estrategia, en su mayoría, manifestaron que se debían incluir aspectos relacionados con la planificación curricular, en lo que respecta a planeamiento, evaluación, recomendaciones, materiales didácticos, distribución del espacio, etc. 


\subsection{Necesidades del personal docente en cuanto a formación continua}

Para conocer las necesidades profesionales que poseen las docentes es necesario conocer acerca de la formación que recibieron en las diferentes universidades. Se pudo observar que fueron pocas las docentes que recibieron contenidos o cursos específicos que abordaran la temática de las agrupaciones heterogéneas; además, se encontró que fue una minoría de la muestra la que realizó alguna práctica con este tipo de agrupación.

Además de la formación universitaria, las docentes deben recibir asesorías y capacitaciones por parte del MEP; sin embargo, tan sólo un $50.41 \%$ de la muestra reveló recibir algún tipo de asesoría por parte del MEP. A pesar de esto, la mayor parte de as docentes consideraban necesaria esta intervención por parte del Ministerio.

Con respecto a las temáticas que las docentes consideran necesarias por tratar en las asesorías, la mayor parte de la muestra manifestó la necesidad de tener más información relacionada con la planificación y la integración de las edades.

\subsection{Principales beneficios y limitaciones de la estrategia}

\section{Beneficios y limitaciones para el desarrollo de las niñas y de los niños}

Entre los beneficios encontrados de esta estrategia para las niñas y los niños fueron la mayor socialización y el desarrollo integral, pues las maestras expresaron que las niñas y los niños eran más sociables y experimentaban una mayor convivencia con sus compañeros, además de aprender nuevas habilidades y nuevos roles. También, destacaron que adquieren mayor seguridad para interactuar, más valores y hábitos.

En cuanto a las limitaciones que tiene la estrategia para el desarrollo del estudiantado, las docentes manifestaron que la diversidad de las edades dificulta el proceso y que las niñas y los niños de transición tienen limitaciones en sus aprendizajes, pues algunas de las maestras tienden a simplificar las actividades para evitar que los menores se sientan frustrados, aburriendo así a los mayores. Es importante que las profesionales sean creativas y propongan actividades lo suficientemente flexibles para poder adaptarlas a todas las edades.

\section{Beneficios y limitaciones para las docentes y los docentes}

Entre los beneficios para las docentes, ellas expresaron que obtenían más experiencia en esta modalidad, además de tener más libertad en su trabajo, al considerar que el planeamiento es más flexible y, en general, sentir que esta estrategia mejora su trabajo. 
Sin embargo, entre las limitaciones que las mismas docentes expresaron estaban la poca preparación que sentían para asumir estos grupos, tal vez producto de poca o ninguna experiencia previa para asumir el trabajo con esta modalidad.

Otra limitación que expresaron fue la dificultad del manejo del grupo, pues decían que como la brecha de edades es amplia, el control de grupo se les dificultaba y esa era una de las razones por las que no realizaban actividades dirigidas a menudo.

Finalmente y pese a todo lo anterior, es notable que todas las categorías son bajas en su porcentaje, siendo muy superior el porcentaje que no responde, lo que nos puede hacer pensar que las docentes no encuentran grandes limitaciones para el trabajo en esta estrategia.

\section{Conclusiones y recomendaciones}

\subsection{Conclusiones}

\section{Características académicas y personales de las docentes}

- Se encontró que, el cuerpo docente ha alcanzado un grado mínimo de Licenciatura en Educación Inicial (77.69\%), lo que indica que están capacitadas para el trabajo en el Nivel Inicial (Ciclo Materno Infantil y Ciclo de Transición).

Cantidad y edades de las niñas y los niños por grupo

- La cantidad de niñas y niños que deben atender las docentes en agrupaciones heterogéneas es acorde con lo estipulado por el MEP en un $60.32 \%$. En general, los grupos no exceden los veinte estudiantes e incluso hay docentes que laboran con grupos menos numerosos.

\section{Funcionalidad del programa de estudio recomendado por el MEP}

- Las docentes manifiestan que se han adaptado sin dificultad a la rutina diaria que el MEP establece, pues les provee la libertad de desarrollar sus propias actividades, ya que las indicadas en el programa son una mera referencia. Sin embargo, la mayoría de las docentes dice tener problemas para elaborar su planeamiento didáctico, ya que las actividades que se programan no responden a las necesidades, e incluso intereses de todo el grupo de niñas y niños. 
- Un $84.30 \%$ de la muestra manifestó la necesidad de que el MEP cree un programa de estudio más adecuado para el trabajo con esta estrategia o modifique el ya existente. Dentro de las modificaciones sugeridas por las docentes tenemos: el planeamiento, pues gran parte de las participantes dice tener dudas, complicaciones, confusiones e incluso problemas a la hora de planear. Algunas elaboran planes para más de quince días, práctica que no responde a los lineamientos dados por el MEP.

- Las actividades propuestas en el Programa de Estudio para el Ciclo Materno Infantil no se plantearon pensando en el trabajo con agrupaciones heterogéneas, de modo que este plan funciona sólo como una guía para la planificación, quedando en las manos del personal docente, en su creatividad y su preparación profesional el éxito de su planificación curricular para atender este tipo de agrupaciones.

- El seguimiento del proceso individual es un espacio donde las niñas y los niños pueden recibir una atención personalizada de acuerdo con sus necesidades y características, permitiéndole al docente seguir la evolución que ellas y ellos tienen con respecto a su desarrollo y a las nuevas necesidades generadas, fortaleciendo no sólo sus áreas intelectuales y físicas, sino también su área emocional. En fin, este tipo de actividad permite una atención integral de las niñas y los niños. Se quiere hacer énfasis en este punto debido a que una cantidad significativa de docentes (el 25.62\%) mostró no incluir dicha actividad en su rutina diaria y, por las razones ya comentadas, esta es de vital importancia en el currículo inicial.

\section{Labor que realiza el personal con la familia y la comunidad}

- Con respecto a la labor que deben desarrollar las docentes con las familias y la comunidad se observa que las participantes tienen presente la importancia de esta labor para la práctica pedagógica, lo cual es una gran ventaja no sólo porque facilita el desarrollo de sus lecciones sino porque, empleando esta estrategia de manera adecuada, se puede beneficiar al estudiante quien tendrá la oportunidad de estar en constante estímulo tanto en la escuela como en el hogar. Además, el acercamiento a la realidad familiar y al entorno permite al docente tener un mayor conocimiento de la realidad en que se desenvuelve cada estudiante, de modo que su labor se torne pertinente y responda a las demandas de la niña y del niño, la familia, la comunidad y la sociedad en general. 
- El acercamiento a la familia se ha dado en mayor medida mediante la invitación a actividades en el aula (91.73\%), reuniones (75.21\%) y actividades extracurriculares $(67.77 \%)$, permitiendo a las docentes conocer la dinámica familiar de cada niña y niño, de modo que el trabajo con esta y la comunidad se verá facilitado pues, por un lado, sabrá cómo trabajar con cada una y cuándo y, por otro, estará estrechamente ligado a la realidad que envuelve al educando dentro de su contexto diario.

\section{Necesidades del personal en cuanto a formación continua}

- Enfocado en el conocimiento de lo que es una agrupación heterogénea, es notorio que la mayoría de las docentes de la muestra posee una vaga definición de lo que es y de las razones de la creación de estas agrupaciones; esto evidencia la necesidad de formar tanto a las docentes que laboran con estas agrupaciones, como a las futuras y a los futuros profesionales en Educación Inicial, de modo que, de ser necesario, estén preparados para asumir estos grupos. Es importante señalar la necesidad de que al trabajar en el Nivel Inicial cuenten con conocimiento no sólo teórico, sino también de contar con la posibilidad de realizar prácticas con estos grupos -durante su formación universitaria-, que permitan a las y los estudiantes acumular mayor experiencia antes de incursionar en el ámbito laboral.

- De acuerdo con la entrevista realizada a las coordinadoras de la carrera de Educación Inicial en cuatro universidades seleccionadas, la visión de estas instituciones es formar profesionales que tengan los conocimientos y las habilidades para desempeñarse con cualquier tipo de estrategia, incluidas las agrupaciones heterogéneas. Además, las docentes de la muestra expresan que uno de los factores que limita su desempeño en esta estrategia es la falta de preparación a nivel universitario para atender este tipo de agrupaciones. De esta manera, se asume que aunque las universidades podrían contemplar -actualmente en sus planes de estudio- el tema de las agrupaciones heterogéneas, esta inclusión es un hecho reciente. Se considera importante resaltar que sólo el $23.97 \%$ de las docentes que conformaron la muestra son egresadas de las universidades tomadas en cuenta para realizar la entrevista con sus coordinadoras de carrera.

- Muchas de las participantes manifestaron la necesidad de tener mejor preparación e incluso orientación en el desarrollo de su práctica profesional; sin embargo, cuando se les preguntó si estaban llevando algún curso o capacitación, el $71.07 \%$ respondió Volumen 9, Número 1, Año 2009, ISSN 1409-4703 
negativamente, lo que deja entrever que, si bien es cierto, tienen necesidades de adquirir mayores conocimientos para desempeñarse de una manera más óptima, son pocas las que siguen en formación. Cabe resaltar que algunas docentes experimentan falta de acceso a las capacitaciones que el MEP imparte, lo que representa un punto importante en cuanto a las posibilidades de formación.

- Las docentes externaron, en repetidas ocasiones, la necesidad imperante de contar no sólo con la oportunidad de continuar capacitándose una vez finalizada su formación universitaria, sino de que se programen de manera constante actividades de diversa índole en las que puedan recibir información y se generen nuevos aprendizajes, además de que puedan compartir sus experiencias, conocimientos e inquietudes con respecto a la labor que desempeñan a diario.

- A pesar de que es tarea de las asesoras regionales brindar asesorías y capacitaciones a las docentes para el trabajo con esta estrategia, el $47.93 \%$ de la muestra indica que no reciben asesorías para desempeñar su trabajo. Además, expresaron que muchas veces estas no responden a sus necesidades reales y están más enfocadas al trabajo en aulas homogéneas.

\section{Principales beneficios y limitaciones del trabajo con agrupaciones heterogéneas}

- Las docentes entrevistadas manifestaron que uno los beneficios de esta estrategia es una mayor posibilidad de integrar a la familia en el proceso educativo. Sin embargo, es importante resaltar que la integración de la familia en la labor de aula no es necesariamente un beneficio exclusivo de esta estrategia, sino que forma parte de los lineamientos para la planificación en todo nivel educativo.

- A raíz de la apertura de estos grupos, se evidencia el compromiso del Estado por brindar una mayor cobertura de la Educación Inicial a nivel nacional. El surgimiento de esta estrategia ha permitido que las niñas y los niños, de zonas rurales dispersas, cuenten con la posibilidad de acceder a una educación desde edades tempranas, beneficiando así su formación integral. De la misma forma, esta estrategia se ha expandido a algunas zonas cercanas al Área Metropolitana, por causas como la baja en la tasa de natalidad y el incremento en la oferta de educación privada a nivel preescolar, lo cual produce una baja Volumen 9, Número 1, Año 2009, ISSN 1409-4703 
matrícula en las instituciones educativas públicas y, por lo tanto, la apertura de nuevos códigos para este tipo de estrategia.

- Un 53.72\% de las docentes indica que en este tipo de agrupaciones existe mayor posibilidad para las niñas y los niños de socializar y convivir entre sí, pues por tratarse de un grupo con diferentes edades, logran un aprendizaje de nuevas habilidades y competencias, facilitando la convivencia y el establecimiento de relaciones afectivas. Esto debido a que se transmiten valores, hábitos y comportamientos de las niñas y de los niños mayores hacia los menores, de modo que desarrollan mayor seguridad. Si se trabaja aprovechando estos beneficios, quienes tengan mayor edad serán modelos para los menores y será posible la transmisión de conductas sociales positivas, puesto que a las niñas y a los niños les gusta ser modelos a imitar y esto favorece valores como la cooperación y el compañerismo.

- Las docentes de la muestra señalan que también existen limitaciones para el trabajo con esta estrategia. Para un $48.75 \%$ de las participantes uno de los más relevantes es el hecho de contar con diversidad de edades en el aula, pues los niños de mayor edad se ven limitados en su aprendizaje debido a que las actividades se simplifican para estar acordes con la edad de los menores. Además, se indica que esto representa una limitante por cuanto los mayores pierden el interés y, si se trata de trabajar a la inversa, los menores se sienten frustrados por la complejidad de las actividades desarrolladas. Es importante señalar que la finalidad de la labor con estos grupos es unificar el trabajo y, de manera integrada, atender las necesidades de todos los implicados en el proceso.

- El $23.97 \%$ de las docentes señala como otras limitantes la falta de control de grupo y la poca preparación que tienen para atenderlos, lo cual puede estar estrechamente ligado con la planificación poco efectiva que se hace de las actividades y rutinas en general. Aunque las docentes indican que los problemas en el manejo de los grupos se debe a la "diferencia de madurez" que tienen las niñas y los niños, es necesario aclarar que estas diferencias se dan en cualquier tipo de agrupación y poco tienen que ver con las edades de estos, sino más bien con su proceso individual de desarrollo. Además, a pesar de que un $85.47 \%$ dice basarse en los intereses y necesidades de las niñas y niños para la planificación el hecho de que tengan problemas para atender este tipo de agrupaciones representa una contradicción, pues si tomaran en cuenta sus intereses y necesidades no deberían haber problemas en Volumen 9, Número 1, Año 2009, ISSN 1409-4703 
cuanto a la efectividad de la planificación y el manejo de aula, pues las actividades y rutinas, en general, captarían la atención del grupo permitiendo el control del mismo.

- El hecho de que ninguna de las docentes señale como beneficio de este tipo de agrupación el que las niñas y los niños mayores ayuden a los menores, hace suponer que ellas no están obteniendo el mayor provecho de las zonas proximales y del andamiaje. Al utilizar esta teoría de la zona de desarrollo próximo, propuesta por Vigotsky, las docentes facilitan su trabajo y las niñas y los niños obtienen un aprendizaje más significativo y eficaz.

- Existen discrepancias entre las opiniones de las educadoras relacionadas con esta estrategia y su trabajo, pues es claro que no todas se sienten a gusto con su labor en estas agrupaciones y se manifiestan insatisfechas en muchas de sus respuestas.

- Un hecho notorio que evidencia la diferencia de opiniones por parte de las docentes de la muestra es que, por un lado, el $28.10 \%$ dice que el trabajo con las familias es mayor, pues se permite integrarlas al proceso, pero, por otro lado, un $19.01 \%$ señala la falta de comunicación con la familia como una de las principales limitantes. Estos señalamientos son del todo contradictorios, de modo que, es necesario indicar que depende del papel que como docente cada una de ellas decida representar y de las estrategias que emplee para lograr que las familias y la comunidad se interesen e incluso se involucren en el proceso educativo.

\subsection{Recomendaciones}

\section{Recomendaciones para el MEP}

- Afianzar la seguridad del cuerpo docente al asumir un grupo heterogéneo, a través de un proceso de inducción o asesoramiento. Dado que las docentes entrevistadas expresaron sentir inseguridad a la hora de realizar su trabajo, lo que podría estar relacionado con la falta de experiencia con este tipo de agrupaciones.

- Garantizar el acceso a las asesorías en diferentes regiones del país. Esto debido a que una de las posibles causas de la falta de formación que expresan tener las docentes que laboran con esta estrategia, es el difícil acceso a centros de estudio, por encontrarse ubicadas en zonas alejadas del Área Metropolitana. 
- Mantener actualizadas a las asesoras regionales de modo que estén preparadas para dar alternativas, sugerencias y guiar, efectivamente, al cuerpo docente en relación al trabajo con agrupaciones heterogéneas, así, las docentes no sentirán evadidas sus dudas.

- Continuar con el criterio de formar grupos no mayores a veinte estudiantes ya que esto favorece el desempeño de las docentes, quienes deben atender a las niñas y a los niños de manera personalizada y procurando así una atención individualizada, de acuerdo con sus necesidades e intereses particulares.

- Crear un nuevo programa de estudio que responda a las características de esta estrategia, procurando la participación del profesorado en su diseño, puesto que éste, al estar en contacto con la realidad educativa y las necesidades de los grupos que atiende, posee una mayor percepción de las necesidades, deficiencias, beneficios y limitaciones que se presentan en su labor.

- Manejar un registro acerca de la ubicación los códigos de grupos heterogéneos. Esto con el fin de facilitar el acceso de particulares e investigadores y los mismos personeros del MEP a dicha información.

\section{Recomendaciones para las universidades}

- Contemplar en los planes de estudio cursos y prácticas relacionadas con grupos heterogéneos. De manera más evidente y constante durante la formación docente, además de ofrecer foros y programas de actualización que contemplen las necesidades del estudiantado -ya egresado- relacionadas con el mercado laboral actual.

- Promover investigaciones sobre la estrategia de grupos heterogéneos.

\section{Recomendaciones para las docentes y los docentes que trabajan con la estrategia}

- Permanecer en constante actualización, a fin de desempeñar una labor con calidad y pertinencia. Ya que con el paso del tiempo hay nuevos estudios, tendencias diversas e innovaciones en la sociedad y, específicamente, en el área de la educación. Cada docente debe preocuparse por obtener, si es necesario por sus propios medios, las herramientas que 
le permitan actualizarse y adquirir diversidad de conocimientos que le darán mayor valor a su trabajo.

- Aplicar eficazmente el conocimiento acerca de la zona de desarrollo próximo, de modo que la experiencia de las niñas y los niños en estas agrupaciones sea más enriquecedora y obtengan un mayor beneficio de su permanencia en esta estrategia.

- El cuerpo docente, al estar en contacto directo con su sujeto de estudio, es quien sabe cuáles son las características, necesidades, técnicas y actividades más adecuadas para el trabajo de aula, por lo que cada docente debe procurar ser investigador y cuestionador de su propia práctica. De tal manera que contribuya al mejoramiento del desempeño profesional, e incluso podrían aportarse nuevos descubrimientos, lo que sería beneficioso para esta estrategia, por tratarse de una práctica novedosa y poco estudiada en nuestro contexto.

- Realizar más investigación acerca de la Estrategia de Grupos Heterogéneos que permitan conocer a cabalidad sus beneficios y limitaciones, así como las técnicas más adecuadas para el desarrollo de las actividades con este tipo de agrupaciones.

\section{Referencias}

Arenas, María Elena; Castro, Eusebio; Gómez, Elia; Hernández, Javier; Méndez, Alejandra; Núñez, Otilia; Sánchez, Leticia; Reyes, Alejandra; Santiago, Irene y Rivas, Martha. (2007). Educación Inicial. Recuperado el día 23 de mayo de 2007, de http://www.lie.upn.mx/docs/MenuPrincipal/LineasEspec/Educlnic.pdf.

Arguedas, Mayra; Bustos, Melba; Chinchilla, Gabriela; González, Carmen; Hutchinson, Sandra y Vargas, Karla. (1999). Propuesta de creación de mecanismos administrativos para mantener excelencia académica de la carrera de Educación Preescolar de la Universidad de Costa Rica. Tesis Licenciatura en Educación Preescolar). Universidad de Costa Rica, San José, Costa Rica.

Berrocal Brenes, Floribeth; Campos Araya, Elena; Sáenz Fernández, Isabel María y Torres Castillo, Carolina.(1993). Evaluación del Plan de Estudios de Bachillerato en Educación Preescolar de la Universidad de Costa Rica. Plan vigente de desde 1989. Seminario de Graduación (Licenciatura en Ciencias de la Educación con énfasis en Educación Preescolar). Universidad de Costa Rica, San José, Costa Rica. 
Blanco García, Sandra; Chaves Castro, Maritza; Corrales Segura, Gladys y Mata Solano, Adelaida.(2002). Implementación Programa de Estudio Ciclo Materno Infantil. Informe Final 2001-2002. San José, Costa Rica.

Bolaños, María José; Cortés, Soledad; Maroto, Andrea; Rojas, Yanncy; Solano, Virginia y Villalobos, Catherine. (2004). La organización didáctica de los grupos heterogé neos y/o mixtos en la Educación Inicial (Preescolar), contemplada desde las necesidades e intereses de los niños(as). Seminario de Graduación (Licenciatura en Educación Preescolar) Universidad de Costa Rica, San José, Costa Rica.

García Llamas, José Luis. (1999). Formación del profesorado. Necesidades y demandas. Barcelona, España: Editorial Praxis.

Katz, Lilian. (2005). Perspectivas Educativas en la Primera Infancia. Santiago de Chile: LOM Ediciones.

Ministerio de Educación Pública. (2004). Programa de Estudio para el Ciclo Materno Infantil. Educación Preescolar. San José, Costa Rica.

Ministerio de Educación Pública (2004). Educación Preescolar en Costa Rica. Consideraciones y lineamientos para el desarrollo de la práctica pedagógica en el nivel Preescolar. San José, Costa Rica.

Ministerio de Educación Pública. (2007). Fascículo para la mediación: fascículo para la familia. Editorial ICER. San José, Costa Rica.

Montero Campos, Elizabeth. (2007). Grupos heterogéneos: Población atendida. Ministerio de Educación Pública. División de Desarrollo Curricular. Departamento de Educación Preescolar. San José. Costa Rica.

Montero Campos, Elizabeth. (2007). Entrevistas personales. Junio de 2007. Departamento de Educación Preescolar, Ministerio de Educación Pública. San José, Costa Rica.

Papalia, Diane, Wendkos, Sally y Duskin, Ruth. (2001). Desarrollo humano. Bogotá, Colombia: McGraw-Hill Interamericana.

Peralta Espinosa, María Victoria. (1988). El currículo en el Jardín Infantil (Un Análisis Crítico). Santiago, Chile: Editorial Andrés Bello.

Picado Vargas, Karina. (2007). El arte de aprender; una guía formativa para el hogar y la escuela. San José, Costa Rica: UNED, La Nación.

Sundell, Knut. (1993). Mixed age groups in Swedish Nursery School and Compulsory School. European Conference on the Quality of Early Childhood Education, 3, 117. Obtenido el 09 de mayo, 2007 de la base de datos global ERIC (EBSCOHost)

Zúñiga León, Irma María (2001). Principios y técnicas para la elaboración de material didáctico para le niño de 0 a 6 años. San José, Costa Rica : EUNED. 


\section{Anexos}

Cuadro 1.Variables de la muestra.

\begin{tabular}{|c|c|}
\hline Variable & Indicadores \\
\hline $\begin{array}{l}\text { 1. Características } \\
\text { académicas y personales } \\
\text { de los docentes }\end{array}$ & $\begin{array}{l}\text { - Sexo } \\
\text { - Edad } \\
\text { - Formación profesional } \\
\text { - Años de experiencia como docente }\end{array}$ \\
\hline $\begin{array}{l}\text { 2. Cantidad y edades de } \\
\text { las niñas y los niños por } \\
\text { grupo }\end{array}$ & $\begin{array}{l}\text { - Cantidad de niñas y niños a cargo del personal } \\
\text { - Cantidad de niñas y niñas según rango de edad }\end{array}$ \\
\hline $\begin{array}{l}\text { 3. Funcionalidad del } \\
\text { programa de estudio } \\
\text { recomendado por el MEP }\end{array}$ & $\begin{array}{l}\text { - Caracterización del espacio de aula (materiales, mobiliario, } \\
\text { dimensiones, iluminación, ventilación) } \\
\text { - Jornada laboral con la que se trabaja } \\
\text { - Estructuración de la rutina } \\
\text { - Planeamiento } \\
\text { - Acciones llevadas a cabo por la docente (instrumentos y técnicas de } \\
\text { evaluación, expedientes) } \\
\text { - Distribución del espacio de aula } \\
\text { - Necesidad de un Programa de Estudios específico para trabajar con la } \\
\text { estrategia heterogénea }\end{array}$ \\
\hline $\begin{array}{l}\text { 4. Labor que realiza el } \\
\text { personal con la familia y la } \\
\text { comunidad }\end{array}$ & $\begin{array}{l}\text { - Visitas al hogar de las niñas y los niños, frecuencia } \\
\text { - Reuniones con familia: frecuencia, temas abordados en estas } \\
\text { - Modo de integrar a la familia en el proceso educativo } \\
\text { - Modo de integrar a la comunidad en el proceso educativo }\end{array}$ \\
\hline $\begin{array}{l}\text { 5. Necesidades del } \\
\text { personal en cuanto a } \\
\text { formación continua }\end{array}$ & $\begin{array}{l}\text { - Conocimiento acerca de la Estrategia de Grupos Heterogéneos } \\
\text { - Formación universitaria para la atención de grupos heterogéneos } \\
\text { - Recomendaciones a las universidades para la fomación de personal } \\
\text { capacitado para el trabajo con grupos heterogéneos } \\
\text { - Asesorias brindadas para el trabajo con grupos heterogéneos }\end{array}$ \\
\hline $\begin{array}{l}\text { 6. Principales beneficios y } \\
\text { limitaciones del trabajo } \\
\text { con agrupaciones } \\
\text { heterogéneas }\end{array}$ & $\begin{array}{l}\text { - Recomendaciones para mejorar la práctica pedagógica en las } \\
\text { agrupaciones heterogéneas } \\
\text { - Beneficios y limitaciones de la Estrategia de Grupos Heterogéneos para } \\
\text { el desarrollo de la niña y del niño, la práctica docente y las familias. }\end{array}$ \\
\hline
\end{tabular}

